\title{
EFEITO DE SUBDOSES DE 2,4-D NA PRODUTIVIDADE DE FUMO E SUSCETIBILIDADE DA CULTURA EM FUNÇÃO DE SEU ESTÁDIO DE DESENVOLVIMENTO ${ }^{1}$
}

\author{
JAMIL CONSTANTIN ${ }^{2}$, RUBENS S. DE OLIVEIRA JÚNIOR ${ }^{2}$, JOSÉ U. T. BRANDÃO \\ FILHO $^{2}$, OSNI CALLEGARI ${ }^{3}$, PAULO H. PAGLIARI ${ }^{4}$, JO $\tilde{O} O$ G. Z. DE ARANTES ${ }^{4}$
}

\begin{abstract}
RESUMO: O presente trabalho teve como objetivo determinar os efeitos de subdoses do herbicida 2,4-D na cultura do fumo. Os experimentos foram conduzidos a campo durante o ano de 2003, no município de Santa Isabel do Ivaí - PR, utilizando-se de fumo tipo Virgínia. No primeiro experimento, foram aplicadas, no estádio de pré-florescimento, doses de 0,$84 ; 1,68 ; 3,36 ; 6,72$; 13,44 e 26,88 g e.a. ha ${ }^{-1}$ de 2,4-D, equivalentes à deriva de 0,$125 ; 0,25 ; 0,5 ; 1,0 ; 2,0$ e 4,0\% de produtos comerciais aplicados a $1,0 \mathrm{~L} \mathrm{ha}^{-1}\left(670 \mathrm{~g}\right.$ e.a. ha $\left.{ }^{-1}\right)$. Para o segundo experimento, os tratamentos foram constituídos pela combinação em esquema fatorial de duas doses $(6,72 \mathrm{e} 13,44 \mathrm{~g}$ e.a. ha $^{-1}$, equivalentes a 1,0 e $2,0 \%$ de deriva) e três épocas de aplicação após o transplante para o campo. Nenhum sintoma visual marcante de fitotoxicidade foi observado dentro da faixa de doses avaliada. Visualmente, alguns dos possíveis efeitos atribuídos à fitotoxicidade ocasionada pela deriva de 2,4-D na cultura do fumo podem estar associados à utilização de antibrotantes. Nenhuma das doses aplicadas no primeiro experimento afetou a produtividade da cultura, nem o aspecto e qualidade das folhas, tendo o fumo tolerado até $4,0 \%$ de deriva avaliada. Resultados obtidos no segundo experimento confirmaram esses dados, constatando-se que níveis de deriva de até 2,0\% $\left(13,44 \mathrm{~g}\right.$ e.a. $\left.\mathrm{ha}^{-1}\right)$, aplicados em três fases após o transplante das mudas para o campo, não causaram qualquer efeito negativo sobre a produtividade da cultura.
\end{abstract}

PALAVRAS-CHAVE: herbicidas fenóxicos, fitotoxicidade, subdoses.

\section{EFFECT OF SUB-LETHAL DOSAGES OF 2,4-D ON TOBACCO YIELD AND CROP SUSCEPTIBILITY AS A FUNCTION OF ITS DEVELOPMENT STAGE}

\begin{abstract}
This work aimed at evaluating the effects of sublethal rates of 2,4-D in tobacco plants. Field experiments were carried out at Santa Isabel do Ivaí (PR) during 2003 in areas cropped with Virginia-type tobacco. For the first trial, plants were sprayed at the pre-flowering stage, at rates of $0.84 ; 1.68 ; 3.36 ; 6.72 ; 13.44$ and $26.88 \mathrm{~g}$ a.e. $\mathrm{ha}^{-1}$ of $2,4-\mathrm{D}$, equivalent to drifts of $0.125 ; 0.25 ; 0.5 ; 1.0 ; 2.0$ and $4.0 \%$ of commercial herbicides applied at $1.0 \mathrm{~L} \mathrm{ha}^{-1}\left(670 \mathrm{~g}\right.$ a.e. ha $\left.{ }^{-1}\right)$. For the second trial, treatments were composed by a factorial combination of two rates $(6.72$ and $13.44 \mathrm{~g}$ a.e. $\mathrm{ha}^{-1}$, equivalent to 1.0 and $2.0 \%$ drifts) and three stages of crop development at the application of 2,4-D. No meaningful visual symptom was observed within the range of rates applied. Visually, some of the eventual effects attributed to phytotoxicity caused by 2,4-D drift to tobacco plants may be associated to sucker suppressing chemicals use in crop. None of the applied rates at first trial provided crop yield or quality damages. Tobacco plants were tolerant up to $4.0 \%$ of 2,4-D simulated drift. Results obtained at second trial agreed with these data, and plants were tolerant up to $2.0 \%$ of simulated drift $\left(13.44 \mathrm{~g}\right.$ a.e. $^{-1} \mathrm{~h}^{-1}$. Rates $\leq 2.0 \%$ applied at three different crop development stages after transplantation to field do not cause any crop yield damage.
\end{abstract}

KEYWORDS: phenoxy herbicides, phytotoxicity, sublethal rates.

\footnotetext{
${ }^{1}$ Trabalho apresentado na forma de pôster no IV International Weed Science Congress (Durban, África do Sul - 20 a 24-6-2004).

${ }^{2}$ Engo Agrônomo, Prof. Adjunto, Departamento de Agronomia, Universidade Estadual de Maringá (UEM), Maringá-PR, Fone: (0XX44) 3261.4407, rsojunior@uem.br, Bolsista CNPq.

${ }^{3}$ Eng $^{\mathrm{o}}$ Agrônomo, M.Sc., Departamento de Agronomia (UEM), Maringá - PR, Bolsista CNPq.

${ }^{4}$ Graduando em Agronomia, Bolsista de Iniciação Científica, área de Ciência das Plantas Daninhas (UEM). Bolsista CNPq. Recebido pelo Conselho Editorial em: 3-3-2005
}

Aprovado pelo Conselho Editorial em: 10-7-2006 


\section{INTRODUÇÃO}

As plantas de fumo são formadas em canteiros de mudas ou em bandejas, onde são semeadas e crescem por aproximadamente 60 dias, quando atingem a altura de 15 a $20 \mathrm{~cm}$. Nesse momento, são retiradas as melhores mudas que são plantadas no campo com raízes nuas. O transplantio é feito nos meses de verão, nas regiões Sudeste e Sul do Brasil (DEUBER, 1997). Trata-se de atividade desenvolvida por pequenos e médios agricultores, sendo os fumicultores, em sua grande maioria, proprietários com área média de 17,9 ha, dos quais apenas 2,5 ha são utilizados para o cultivo do fumo. A área média cultivada por família é de 2,2 hectares.

A cultura do fumo encontra-se basicamente concentrada na região Sul do Brasil, a qual responde por $96,4 \%$ do total produzido no País (AFUBRA, 2004). Nessa região, são produzidos fumos claros, destinados exclusivamente para o fabrico de cigarros, enquanto, no Nordeste, são produzidos fumos escuros, próprios para a fabricação de charutos e cigarrilhas. O principal tipo de fumo produzido na região Sul é o Virgínia, seguido do Burley. O fumo Comum é um fumo produzido exclusivamente para o mercado doméstico. A produção dos tipos Virgínia e Burley, consideradas variedades nobres, além de atender à demanda interna, destina-se ao mercado externo.

Em alguns estados brasileiros, tem se levantado a polêmica em torno da proibição ou limitação do uso do 2,4-D, herbicida do grupo químico dos ácidos fenóxicos, em função dos problemas que ocorrem pela deriva do produto. No entanto, poucos trabalhos têm mostrado a dimensão desse problema do ponto de vista dos possíveis danos que podem ser causados às culturas sensíveis. Há suspeitas de que o fumo esteja entre as culturas sensíveis e que o contato da planta com baixas concentrações do 2,4-D, oriundas de derivas, possa injuriar a cultura. No entanto, a revisão bibliográfica demonstra que não existem dados que subsidiem tal conclusão.

O objetivo deste trabalho foi determinar a intensidade de danos causados à produção da cultura do fumo pela aplicação de subdoses de 2,4-D, reproduzindo a ocorrência de deriva, assim como determinar o nível de resíduos nas folhas que eventualmente tenham entrado em contato com tais níveis desse herbicida.

\section{MATERIAL E MÉTODOS}

Os experimentos foram conduzidos no campo, durante o ano de 2003, no município de Santa Isabel do Ivaí - PR.

As mudas de fumo foram produzidas em bandeja "float" (substrato Plantmax) e, quando estavam com aproximadamente 60 dias, foram transplantadas para o campo. Foi utilizado o fumo tipo Virgínia, com espaçamento de 0,5 m entre plantas da mesma fileira e 1,20 m entre fileiras. A adubação de transplante consistiu de $36 \mathrm{~g}$ do formulado 10-18-20 por planta. Em cobertura, foram aplicados $20 \mathrm{~g}$ de salitre $\left(\mathrm{KNO}_{3}\right)$ por planta, aos 20 dias após o transplante, e $10 \mathrm{~g}$ planta $^{-1}$ aos 40 dias após o transplante. Os demais tratos culturais foram feitos segundo aqueles normalmente recomendados pela assistência técnica (FELIPE, 1996).

No primeiro experimento, os tratamentos utilizados foram constituídos pela aplicação das doses de 0,$84 ; 1,68 ; 3,36 ; 6,72 ; 13,44$ e 26,88 g de equivalente ácido (e.a.) de 2,4-D por hectare, além das testemunhas duplas sem herbicida. Em condições de campo, as doses utilizadas no experimento equivaleriam à deriva de 0,$125 ; 0,25 ; 0,5 ; 1,0 ; 2,0$ e $4,0 \%$ dos produtos comerciais mais comuns, aplicados a $1,0 \mathrm{~L} \mathrm{ha}^{-1}\left(670 \mathrm{~g}\right.$ e.a. ha $\left.{ }^{-1}\right)$, a qual é uma dose usualmente utilizada no manejo de áreas antecedendo a semeadura. Em todos os tratamentos com herbicida, acrescentou-se Agral a $0,1 \%$ v/v (adesivo).

O método de testemunhas duplas foi desenvolvido com o intuito de aferir com maior precisão experimentos na área de Ciência das Plantas Daninhas, sendo bastante útil em trabalhos relacionados à avaliação da tolerância de culturas a herbicidas e de matointerferência. A metodologia encontra-se descrita em detalhes em publicações recentes (CONSTANTIN et al., 2001; FAGLIARI et al., 2001; MESCHEDE et al., 2004). 
Para as aplicações, utilizou-se de pulverizador costal de pressão constante, pressurizado a $\mathrm{CO}_{2}$, à pressão de $207 \mathrm{kPa}$, equipado com pontas de jato plano tipo leque XR 11002, proporcionando volume de calda equivalente a $200 \mathrm{~L} \mathrm{ha}^{-1}$.

As aplicações foram realizadas 30 dias após o transplante das mudas para o campo, quando as plantas se encontravam no estádio de pré-florescimento, tendo, em média, 28 folhas e 1,25 $\mathrm{m}$ de altura. No momento da aplicação, o solo apresentava-se úmido, a temperatura estava em $29{ }^{\circ} \mathrm{C}$ e a umidade relativa do ar em $80 \%$. Em todas as aplicações, foram utilizadas cortinas protetoras para cada parcela, evitando-se qualquer deriva para as parcelas vizinhas. A colheita foi realizada escalonadamente, à medida que as folhas amadureciam, e iniciou-se 45 dias após o transplante das mudas para o campo.

Foi utilizado o delineamento em blocos ao acaso, com quatro repetições. Para efeito de discussão dos dados, realizou-se comparação entre cada tratamento e sua respectiva testemunha. Portanto, para a análise dos resultados, utilizou-se apenas do teste $\mathrm{F}$, uma vez que o objetivo do trabalho foi avaliar o grau de injúria de cada tratamento à produtividade da cultura.

Foram coletadas amostras de folhas (12 folhas por planta, em cinco plantas por parcela experimental) para a análise de resíduos. Após a coleta e até o processamento em laboratório, as amostras foram conservadas em freezer $\left(-16{ }^{\circ} \mathrm{C}\right)$. Foram utilizados $10 \mathrm{~g}$ de amostra previamente moído e homogeneizada em frasco Schott de extração. Adicionaram-se $50 \mathrm{~mL}$ de extrator metanol alcalino (Metanol/ $\mathrm{NaOH} 20 \%$ ), passando a seguir as amostras no polytron por 30 segundos. As amostras foram agitadas por $2 \mathrm{~h}$ e centrifugadas por 5 minutos a $2.500 \mathrm{rpm}$. Pipetou-se uma alíquota de $10 \mathrm{~mL}$ da solução, a qual foi evaporada por aproximadamente 90 minutos no turboevaporador a $35{ }^{\circ} \mathrm{C}$. Adicionaram-se $10 \mathrm{~mL}$ de água deionizada, $4 \mathrm{~g}$ de cloreto de sódio e $2 \mathrm{~mL}$ de ácido sulfúrico $6 \mathrm{~mol} \mathrm{~L}^{-1}$. A partição foi realizada em $10 \mathrm{~mL}$ de éter etílico, seguindo-se de agitação vigorosa, sendo as amostras centrifugadas por 3 minutos a $900 \mathrm{rpm}$. A fase éter foi recolhida em tubos de centrífuga e evaporada a $35^{\circ} \mathrm{C}$. Adicionaram-se $3 \mathrm{~mL}$ de metanol aos tubos, os quais foram colocados no ultra-som por 10 segundos. Colunas de alumina foram preparadas com $5 \mathrm{~mL}$ de metanol, carregando-as posteriormente com o extrato, o qual foi eluído com ácido sulfúrico $1 \mathrm{~mol} \mathrm{~m}^{-3}$. O eluto foi recolhido em tubo de centrífuga, acrescentando a este $4 \mathrm{~g}$ de cloreto de sódio e $10 \mathrm{~mL}$ de éter etílico, agitado vigorosamente e centrifugado por 3 minutos a $900 \mathrm{rpm}$. O éter foi transferido para outro tubo e evaporado novamente. Adicionou-se $0,5 \mathrm{~mL}$ de ácido sulfúrico $1 \mathrm{~mol} \mathrm{~m}^{-3} \mathrm{em}$ n-propanol, levando os tubos novamente ao ultra-som e depois ao bloco aquecedor por 30 minutos a $100{ }^{\circ} \mathrm{C}$. Após a retirada do bloco, as amostras receberam $2 \mathrm{~mL}$ de água deionizada e $2 \mathrm{~mL}$ de solução do padrão interno $\left(0,2 \mu \mathrm{g} \mathrm{mL} \mathrm{m}^{-1}\right.$ picloram P.E. em hexano). As amostras foram novamente centrifugadas como anteriormente, e $4 \mu \mathrm{L}$ da amostra foram injetados no cromatógrafo de fase gasosa, modelo HP 5890, equipado com detector seletivo de massa (GC/MSD). As condições de funcionamento do cromatógrafo foram as seguintes: temperatura inicial de $70{ }^{\circ} \mathrm{C}$, com elevação de $20{ }^{\circ} \mathrm{C} \min ^{-1}$ até $300{ }^{\circ} \mathrm{C}$ por 4 minutos; detector: $280{ }^{\circ} \mathrm{C}$; injetor: $270{ }^{\circ} \mathrm{C}$; tempo de análise: 16,5 minutos. Para o fumo, o método proporcionou limite de detecção de $0,050 \mathrm{mg} \mathrm{kg}^{-1}$, com recuperação média de $76 \pm 4 \%$. As análises de resíduos foram realizadas apenas para o primeiro experimento.

Para o segundo experimento, os tratamentos foram constituídos pela combinação em esquema fatorial de duas doses $\left(6,72\right.$ e $13,44 \mathrm{~g}$ e.a. ha ${ }^{-1}$ equivalentes a derivas de 1,0 e $2,0 \%$ da dose comercialmente utilizada do produto) e três épocas de aplicação, com a intenção de avaliar se a suscetibilidade do fumo ao 2,4-D poderia ser influenciada pelo estádio de desenvolvimento da cultura. A primeira aplicação foi realizada em 13-10-2003, quando o fumo possuía, em média, 17,5 folhas e $55 \mathrm{~cm}$ de altura; a segunda em 23-10-2003 (fumo com 21 folhas e $80 \mathrm{~cm}$ de altura) e a terceira em 6-11-2003 (fumo com 25 folhas e 1,20 m de altura). Foram adotados os mesmos procedimentos do primeiro experimento com relação ao isolamento das parcelas, número de repetições, delineamento experimental e avaliação da produtividade. 


\section{RESULTADOS E DISCUSSÃO}

Nenhum sintoma visual marcante de fitointoxicação foi observado dentro da faixa de doses avaliada. Aparentemente, alguns dos possíveis efeitos atribuídos à fitotoxicidade ocasionada pela deriva de 2,4-D para a cultura do fumo podem estar associados à utilização de antibrotantes, utilizados na "capação" química, usual na cultura. Nenhuma das doses aplicadas afetou a produtividade de folhas da cultura (Tabela 1), nem seu aspecto e qualidade. Tais constatações indicam que o fumo é mais tolerante ao 2,4-D do que previamente reportado, ao menos após o transplantio para o campo. Análises realizadas em laboratório (GC/MS), em amostras de folhas coletadas por ocasião da colheita, não apresentaram níveis de resíduos detectáveis (Tabela 2) dentro do limite de quantificação do método empregado $(0,050 \mathrm{mg} \mathrm{kg}$ ). De acordo com WAUCHOPE et al. (1992), a meia-vida do 2,4-D, em condições de campo, é de aproximadamente 10 dias, o que poderia explicar a não-detecção da molécula nas análises de resíduos realizadas.

TABELA 1. Produtividade de folhas de fumo Virgínia em função de doses de 2,4-D aplicadas, simulando deriva na época de pré-florescimento. Média de quatro repetições.

\begin{tabular}{|c|c|c|}
\hline \multirow{2}{*}{$\begin{array}{c}\left.\text { Dose (g e.a. ha }{ }^{-1}\right) \text { e nível de deriva estimado } \\
\text { em relação à dose de } 1 \mathrm{~L} \text { ha das formulações } \\
\text { comerciais }(\%)\end{array}$} & \multicolumn{2}{|c|}{ Produtividade de Folhas $\left(\mathrm{t} \mathrm{ha}^{-1}\right)$} \\
\hline & Na parcela tratada & $\begin{array}{l}\text { Na respectiva testemunha } \\
\text { sem herbicida }\end{array}$ \\
\hline $0,84(0,125 \%)$ & $26,26 \mathrm{a}$ & $25,88 \mathrm{a}$ \\
\hline $1,68(0,25 \%)$ & $25,13 \mathrm{a}$ & $28,80 \mathrm{a}$ \\
\hline $3,36(0,50 \%)$ & 25,46 a & $23,13 \mathrm{a}$ \\
\hline $6,72(1,00 \%)$ & $25,92 \mathrm{a}$ & $22,88 \mathrm{a}$ \\
\hline $13,44(2,00 \%)$ & $23,82 \mathrm{a}$ & $25,82 \mathrm{a}$ \\
\hline $26,88(4,00 \%)$ & $23,73 \mathrm{a}$ & $24,95 \mathrm{a}$ \\
\hline
\end{tabular}

Médias seguidas por mesma letra nas linhas não diferem entre si, pelo teste $\mathrm{F}$ (5\% de probabilidade).

TABELA 2. Análise de resíduos de 2,4-D em folhas de fumo colhidas após a aplicação de diferentes doses no estádio de pré-florescimento.

\begin{tabular}{cc}
\hline $\begin{array}{c}\text { Dose }(\mathrm{g} \text { e.a. ha } \\
\text { dose de } 1 \mathrm{~L} \mathrm{ha}^{-1} \text { dável de deriva estimado em relação à }\end{array}$ & ${\text { Resíduo }(\mathrm{mg} \mathrm{kg})^{-1}}^{-1}$ \\
\hline $0,84(0,125 \%)$ & N.D.* \\
$1,68(0,25 \%)$ & N.D. \\
$3,36(0,50 \%)$ & N.D. \\
$6,72(1,00 \%)$ & N.D. \\
$13,44(2,00 \%)$ & N.D. \\
$26,88(4,00 \%)$ & N.D. \\
Testemunha sem herbicida & N.D. \\
\hline
\end{tabular}

*N.D. = Nada detectável.

Resultados obtidos no segundo experimento confirmaram esses dados, constatando-se que níveis de deriva de até 2,0\% (13,44 g e.a.ha $\left.{ }^{-1}\right)$, aplicados em três fases mais precoces do que aquelas utilizadas para o primeiro experimento, não causaram qualquer efeito negativo sobre a produtividade da cultura (Tabela 3). Esse fato demonstra que há pouca variação na tolerância das plantas em função do estádio de desenvolvimento após o transplantio para o campo. Tal constatação implica o fato de que se pode considerar o fumo como pouco sensível a subdoses de 2,4-D, comparativamente a outras culturas.

As respostas de espécies de plantas à aplicação de subdoses de 2,4-D podem variar desde a completa seletividade até a elevada suscetibilidade. A cultura da canola, por exemplo, pode ser considerada sensível, uma vez que há redução na produtividade mesmo quando a cultura é exposta à aplicação de dose equivalente a apenas $1,5 \%$ da comercialmente utilizada (WALL, 1996). Por outro lado, SCHOROEDER et al. (1983) avaliaram a deriva simulada do herbicida 2,4-D sobre a cultura da beterraba (Beta vulgaris L.), em diferentes estádios de desenvolvimento da cultura, 
aplicando doses equivalentes a 35; 140 e $280 \mathrm{~g}$ e.a.ha ${ }^{-1}$. As aplicações foram realizadas nos estádios 1 (3-4 folhas), 2 (6-8 folhas), 3 (15-20 folhas), 4 (20-25 folhas) e 5 (30-35 folhas). A dose de $35 \mathrm{~g}$ e.a. ha ${ }^{-1}$, independentemente do estádio de aplicação, não afetou a produtividade, a pureza e o teor de sacarose da cultura, quando comparado com a testemunha. De acordo com os autores, esses dados sustentariam a teoria de que doses relativamente pequenas de 2,4-D podem até causar discreto incremento na produtividade da beterraba. As doses de 140 e $280 \mathrm{~g}$ e.a.ha ${ }^{-1}$, aplicadas nos dois primeiros estádios da cultura, reduziram a produtividade.

TABELA 3. Produtividade de folhas de fumo Virgínia em função de doses de 2,4-D em diferentes estádios de desenvolvimento da cultura do fumo. Média de quatro repetições.

\begin{tabular}{lcc}
\hline \multirow{2}{*}{ Estádio e Dose $\left(\mathrm{g}\right.$ e.a. $\left.\mathrm{ha}^{-1}\right)$} & \multicolumn{2}{c}{ Produtividade $\left(\mathrm{kg} \mathrm{ha}^{-1}\right)$} \\
\cline { 2 - 3 } Estádio $80 \mathrm{~cm}$ - dose $6,72(1,0 \%)$ & Na parcela tratada & Na respectiva testemunha sem herbicida \\
Estádio $80 \mathrm{~cm}$ - dose $13,44(2,0 \%)$ & $18,96 \mathrm{a}$ & $17,83 \mathrm{a}$ \\
Estádio $100 \mathrm{~cm}$ - dose 6,72(1,0\%) & $17,64 \mathrm{a}$ & $18,18 \mathrm{a}$ \\
Estádio $100 \mathrm{~cm}$ - dose $13,44(2,0 \%)$ & $17,68 \mathrm{a}$ & $18,17 \mathrm{a}$ \\
Estádio $120 \mathrm{~cm}$ - dose 6,72 $(1,0 \%)$ & $18,66 \mathrm{a}$ & $18,08 \mathrm{a}$ \\
Estádio $120 \mathrm{~cm}$ - dose $13,44(2,0 \%)$ & $18,30 \mathrm{a}$ & $17,64 \mathrm{a}$ \\
\hline
\end{tabular}

Dentro da mesma linha, médias seguidas por mesma letra não diferem entre si, pelo teste $\mathrm{F}$ ( $5 \%$ de probabilidade).

\section{CONCLUSÕES}

O fumo Virgínia demonstrou baixa sensibilidade a subdoses de 2,4-D, tolerando concentrações equivalentes a 4,0\%.

O estádio de desenvolvimento da cultura não influenciou na sensibilidade ao 2,4-D, dentro da faixa de concentrações utilizada.

\section{REFERÊNCIAS}

AFUBRA. Associação dos Fumicultores do Brasil. Produção de fumo por tipo. Disponível em: $<$ http://www.afubra.com.br/institucional/index.php?idiomas_id=1\&acao=conteudo\&conteudos_id= 17>. Acesso em: 6 set. 2004.

CONSTANTIN, J.; OLIVEIRA JÚNIOR, R.S.; FAGLIARI, J.R. Emprego de testemunhas duplas adjacentes na avaliação da seletividade de herbicidas aplicados na cultura do milho. Varia Scientia, Cascavel, v.1, n.2, p.61-74, 2001.

DEUBER, R. Ciência das plantas infestantes. Manejo. Campinas: [s.n.], 1997. v.2. p.117-18.

FAGLIARI, J.R.; OLIVEIRA JÚNIOR, R.S.; CONSTANTIN, J. Métodos de avaliação da seletividade de herbicidas para a cultura da cana-de-açúcar. Acta Scientiarum, Maringá, v.23, n.5, p.1229-34, 2001.

FELIPE, J.M. Tratos culturais: pragas, doenças, colheita e armazenamento da cultura do fumo (Nicotiana tabacum L.). In: BRINHOLI, O. Cultura do fumo. Botucatu: UNESP, 1996. p.41-92.

MESCHEDE, D.K.; OLIVEIRA JÚNIOR, R.S.; CONSTANTIN, J.; SCAPIM, C.A. Período anterior à interferência em soja: estudo de caso com baixa densidade de estande e testemunhas duplas. Planta Daninha, Viçosa, MG, v.22, n.2, p.239-46, 2004.

SCHOROEDER, G.L.; COLE, D.F.; DEXTER, A.G. Sugarbeet (Beta vulgaris L.) response to simulated herbicide spray drift. Weed Science, Champaign, v.31, n.6, p.831-6, 1983.

WALL, D.A. Effect of sublethal dosages of 2,4-D on annual broadleaf crops. Canadian Journal of Plant Science, Ottawa, v.76, n.1, p.179-85, 1996.

WAUCHOPE, R.D.; BUTTLER, T.M.; HORNSBY, A.G.; AUGUSTIJN-BECKERS, P.W.M.; BURT, J.P. The SCS/ARS/CES pesticide properties database for environmental decision-making. Reviews of Environmental Contamination Toxicology, New York, v.123, p.1-164,1992. 